\title{
SOBRE UMA EXTENSÃO DE CÁLCULO ESPINORIAL (I)
}

\author{
Mario Schönberg
}

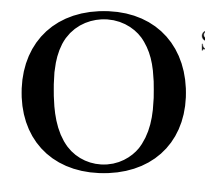

S ESPINORES foram introduzidos para obter todas as representações lineares irredutíveis do grupo das rotações e reviramentos de um espaço euclidiano. Algumas dessas representações já são dadas pelo cálculo tensorial, mas existem outras já conhecidas há muito para o espaço tridimensional e o espaço de Minkowski. Estudando as representações lineares dos grupos simples e semi-simples, Cartan construiu, de um ponto de vista algébrico-analítico, todas as representações irredutíveis e mostrou a existência dos espinores. Esse fato despertou pouca atenção até a descoberta da equação de Dirac para o elétron. Darwin observou que essa equação é relativisticamente invariante apesar das componentes da função de onda não formarem um quadrivetor. Recentemente, Cartan estudou uma representação geométrica para um tipo particular de espinor, por ele denominado de espinor simples, e mostrou como os números hipercomplexos de Clifford-Lipschitz podem ser introduzidos de um modo muito natural.

Na presente série de trabalhos abordaremos o problema de uma teoria geométrica dos espinores sob um ponto de vista diferente do de Cartan. Relacionamos o espinor com as coordenadas projetivas sobre o absoluto do espaço euclidiano considerado. Somos assim conduzidos naturalmente a estender o conceito de espinor aos espaços não euclidianos, em que também podemos introduzir um absoluto seguindo os métodos de Cayley e Klein. O grupo conforme de um espaço euclidiano sendo isomorfo ao grupo de rotações e reviramentos de um espaço não euclidiano oportuno obtemos imediatamente uma extensão do cálculo espinorial do grupo conforme de um espaço euclidiano. 


\section{Deslocamentos e reviramentos euclidianos como projetividades}

1. Recordaremos rapidamente as relações entre a teoria dos deslocamentos e reviramentos e a geometria projetiva. Essas transformações sendo contínuas, algébricas e biunívocas e transformando planos em planos são homografias. Mais precisamente são equiafinidades porque conservam o plano do infinito e tem determinante \pm 1 porque conservam os volumes.

Os deslocamentos e reviramentos conservam as distâncias e portanto transformam retas isotropas em retas isotropas e cones isotropos em cones isotropos. Conservando o plano do infinito e transformando cones isotropos em cones isotropos conservam o absoluto, intersecção dos cones isotropos com o plano do infinito. Obtemos assim a propriedade fundamental dos deslocamentos e reviramentos de serem projetividades que conservam o absoluto. Reciprocamente se demonstra facilmente que qualquer projetividade que conserva o absoluto é uma semelhança, os deslocamentos e reviramentos são simplesmente as semelhanças de razão \pm 1 .

Analisemos as homografias que as semelhanças induzem sobre o absoluto. Introduzindo uma coordenada projetiva $\lambda$ sobre o absoluto, as projetividades sobre o absoluto serão dadas por transformações lineares fracionárias de $\lambda$ :

$$
\lambda=\frac{\alpha \lambda^{\prime}+\beta}{\gamma \lambda^{\prime}+\delta}
$$

ou homogeneizando o parâmetro $\lambda=\frac{\lambda_{1}}{\lambda_{2}}$

$$
\left\{\begin{array}{l}
\lambda_{1}=\alpha \lambda_{1}^{\prime}+\beta \lambda_{2}^{\prime} \\
\lambda_{2}=\gamma \lambda_{1}^{\prime}+\delta \lambda_{2}^{\prime}
\end{array},\right.
$$

Geometricamente a introdução do parâmetro pode ser feita tomando uma esfera e considerando as duas famílias de geratrizes retilíneas que são retas isotropas. Por cada ponto do absoluto passa uma e só uma reta de cada família e $\lambda$ pode ser tomado como o parâmetro da geratriz de uma das famílias que passa pelo ponto considerado do absoluto. A natureza da homografia sobre o absoluto depende da natureza da matriz da substituição (2): 


$$
H=\left(\begin{array}{l}
\alpha \beta \\
\gamma \delta
\end{array}\right)
$$

Convém reduzir $H$ à forma canônica. Para isso observemos que uma semelhança resulta de uma homotetia seguida de um deslocamento. Tomando a origem no centro de homothetia a transformação de coordenadas que lhe corresponde é da forma:

$$
\begin{aligned}
& x^{\prime \prime}=k x \\
& y^{\prime \prime}=k y \\
& z^{\prime \prime}=k z
\end{aligned}
$$

Tomando o eixo dos z na direção do eixo de rotação do deslocamento, a transformação das coordenadas que lhe corresponde é:

$$
\begin{aligned}
x^{\prime} & =a+x^{\prime \prime} \cos \theta+y^{\prime \prime} \operatorname{sen} \theta \\
y^{\prime} & =b-x^{\prime \prime} \operatorname{sen} \theta+y^{\prime \prime} \cos \theta \\
z^{\prime} & =c+z^{\prime \prime}
\end{aligned}
$$

donde finalmente:

$$
\begin{aligned}
& x^{\prime}=a+k(x \cos \theta+y \operatorname{sen} \theta) \\
& y^{\prime}=b-k(x \operatorname{sen} \theta-y \cos \theta \\
& z^{\prime}=x+k z
\end{aligned}
$$

ou em coordenadas homogêneas:

$$
\begin{aligned}
& \rho x_{1}^{\prime}=a x_{4}+k\left(x_{1} \cos \theta+x_{2} \operatorname{sen} \theta\right) \\
& \rho x_{2}^{\prime}=b x_{4}-k\left(x_{1} \operatorname{sen} \theta-x_{2} \cos \theta\right) \\
& \rho x_{3}^{\prime}=c x_{4}+k x_{3} \\
& \rho x_{4}^{\prime}=x_{4} .
\end{aligned}
$$


A coordenada projetiva pode ser introduzida como segue:

$$
\begin{array}{cc}
\sigma\left(x_{1}+i x_{2}\right)=\lambda_{1}^{2} & \sigma\left(x_{1}-i x_{2}\right)=-\lambda_{2}^{2} \\
\sigma x_{3}=\lambda_{1} \lambda_{2} & \sigma x_{4}=0 \\
\sigma^{2}\left(x_{1}^{2}+x_{2}^{2}+x_{3}^{2}\right)=-\lambda_{1}^{2} \lambda_{2}^{2}+\left(\lambda_{1} \lambda_{2}\right)^{2}=0 .
\end{array}
$$

À transformação (3d) dos $x$ corresponde a transformação seguinte $\operatorname{dos} \lambda$ :

$$
\begin{aligned}
& \tau \lambda_{1}^{2}=k c^{+i \theta} \lambda_{1}^{\prime 2} \\
& \tau \quad \lambda_{2}^{2}=k c^{-i \theta} \lambda_{2}^{\prime 2} \\
& \tau \quad \lambda_{1} \lambda_{2}=k \lambda_{1}^{\prime} \lambda_{2}^{\prime} \\
& \lambda=c^{i \theta} \lambda^{\prime}
\end{aligned}
$$

A formula (6) mostra que as semelhanças induzem sobre o absoluto projetividades parabólicas na hipótese de $\theta$ não ser múltiplo de $2 \pi$, caso em que a projetividade sobre o absoluto, se reduz à identidade. Veremos em seguida o que representam as projetividades não parabólicas do absoluto.

\section{Projetividades sobre uma espera real}

3. O estudo das rotações e reviramentos em torno de um ponto fixo pode ser feito de modo conveniente pela análise das projetividades sobre a esfera de raio 1 , com centro no centro das rotações.

As rotações e reviramentos em torno de um ponto fixo induzem projetividades sobre os dois sistemas de geratrizes retilíneas das esferas com o centro nesse ponto. As projetividades não degeneradas sobre uma esfera são de dois tipos: as do primeiro conservam os dois sistemas de geratrizes retilíneas e as do segundo trocam os dois sistemas. Com efeito as duas geratrizes que passam pelo ponto $P$ se transformam nas duas geratrizes que passam por $P^{\prime}$, isso mostra que geratrizes de sistemas diferentes são transformadas em geratrizes de sistemas diferentes. Ora todas as geratrizes 
do sistema I encontram uma geratriz qualquer do sistema II e assim o sistema I é transformado no sistema que contém a transformada da geratriz II considerada, o que demonstra nossa afirmação.

Tomando o centro de rotação como origem de um sistema de coordenadas cartesianas ortogonais as equações dos dois sistemas de geratrizes podem ser postas sob a forma:

$$
\begin{gathered}
\left\{\begin{array}{l}
\lambda_{1}\left(x_{3}-x_{4}\right)+\lambda_{2}\left(x_{1}+i x_{2}\right)=0 \\
\lambda_{1}\left(x_{1}-i x_{2}\right)-\lambda_{2}\left(x_{3}+x_{4}\right)=0
\end{array}\right. \\
\left\{\begin{array}{l}
\mu_{1}\left(x_{3}-x_{4}\right)+\mu_{2}\left(x_{1}+i x_{2}\right)=0 \\
\mu_{1}\left(x_{1}-i x_{2}\right)-\mu_{2}\left(x_{3}+x_{4}\right)=0
\end{array}\right.
\end{gathered}
$$

As coordenadas de um ponto qualquer da esfera podem ser representadas parametricamente em função dos parâmetros das geratrizes que passam por esse ponto; as fórmulas são:

$$
\left\{\begin{array}{l}
\rho x_{1}=\lambda_{1} \mu_{2}+\mu_{1} \lambda_{2} \\
\rho x_{2}=-i\left(\lambda_{1} \mu_{2}-\lambda_{2} \mu_{1}\right) \\
\rho x_{3}=\lambda_{1} \mu_{1}-\lambda_{2} \mu_{2} \\
\rho x_{4}=\lambda_{1} \mu_{1}+\lambda_{2} \mu_{2}
\end{array}\right.
$$

As fórmulas (4) são um caso particular de (8), pois a esfera considerada passa pelo absoluto. Vemos assim que a introdução do parâmetro projetivo sobre o absoluto feita pelas fórmulas (4) é bem a tradução algébrica do método geométrico anteriormente proposto.

Uma projetividade do primeiro tipo induz sobre os dois sistemas de geratrizes transformações dadas pelas fórmulas:

$$
\left\{\begin{array} { l } 
{ \lambda _ { 1 } = \alpha \lambda _ { 1 } ^ { \prime } + \beta \lambda _ { 2 } ^ { \prime } } \\
{ \lambda _ { 2 } = \gamma \lambda _ { 1 } ^ { \prime } + \delta \lambda _ { 2 } ^ { \prime } } \\
{ \alpha \delta - \beta \gamma \neq 0 }
\end{array} \quad ( 9 \mathrm { a } ) \quad \left\{\begin{array}{l}
\mu_{1}=a \mu_{1}^{\prime}+b \mu_{2}^{\prime} \\
\mu_{2}=c \mu_{1}^{\prime}+d \mu_{2}^{\prime} \\
a d-b c \neq 0
\end{array}\right.\right.
$$


Para uma projectividade do segundo tipo temos:

$$
\left\{\begin{array} { l } 
{ \mu _ { 1 } = \alpha \lambda _ { 1 } ^ { \prime } + \beta \lambda _ { 2 } ^ { \prime } } \\
{ \mu _ { 2 } = \gamma \lambda _ { 1 } ^ { \prime } + \delta \lambda _ { 2 } ^ { \prime } }
\end{array} \quad ( 1 0 \mathrm { a } ) \quad \left\{\begin{array}{l}
\lambda_{1}=a \mu_{1}^{\prime}+b \mu_{2}^{\prime} \\
\lambda_{2}=c \mu_{1}^{\prime}+d \mu_{2}^{\prime}
\end{array}\right.\right.
$$

Uma projetividade do primeiro tipo pode sempre ser obtida como produto de duas projetividades que conservam fixas as geratrizes de um sistema:

$$
\begin{array}{ll} 
\begin{cases}\lambda_{1}^{\prime \prime}=\alpha \lambda_{1}^{\prime}+\beta \lambda_{2}^{\prime} \\
\lambda_{2}^{\prime \prime}=\gamma \lambda_{1}^{\prime}+\delta \lambda_{2}^{\prime}\end{cases} \\
\left\{\begin{array}{l}
\lambda_{1}=\lambda_{1}^{\prime \prime} \\
\lambda_{2}=\lambda_{2}^{\prime \prime}
\end{array}\right. & \left\{\begin{array}{l}
\mu_{1}^{\prime \prime}=\mu_{1}^{\prime} \\
\mu_{2}^{\prime \prime}=\mu_{2}^{\prime}
\end{array}\right. \\
\end{array}
$$

As matrizes $\left(\begin{array}{ll}\alpha & \beta \\ \gamma & \delta\end{array}\right)$ e $\left(\begin{array}{ll}a & b \\ c & d\end{array}\right)$ são imaginárias conjugadas de modo a que os sistemas transformados também sejam conjugados.

Uma decomposição análoga pode ser feita para as projetividades do segundo tipo:

$$
\begin{array}{ll} 
\begin{cases}\lambda_{1}^{\prime \prime}=\alpha \lambda_{1}^{\prime}+\beta \lambda_{2}^{\prime} \\
\lambda_{2}^{\prime \prime}=\gamma \lambda_{1}^{\prime}+\delta \lambda_{2}^{\prime}\end{cases} \\
\left\{\begin{array}{l}
\lambda_{1}^{\prime \prime \prime}=\lambda_{1}^{\prime \prime} \\
\lambda_{2}^{\prime \prime \prime}=\lambda_{2}^{\prime \prime}
\end{array}\right. \\
\left\{\begin{array}{l}
\lambda_{1}=\mu_{1}^{\prime \prime \prime} \\
\lambda_{2}=\mu_{2}^{\prime \prime \prime}
\end{array}\right. & \left\{\begin{array}{l}
\mu_{1}^{\prime \prime}=\mu_{1}^{\prime} \\
\mu_{2}^{\prime \prime}=\mu_{2}^{\prime \prime \prime}=a \mu_{1}^{\prime \prime}+b \mu_{2}^{\prime \prime}
\end{array}\right. \\
\mu_{2}^{\prime \prime \prime}=c \mu_{1}^{\prime \prime}+d \mu_{2}^{\prime \prime}
\end{array}
$$

Apliquemos essas considerações no caso das projetividades induzidas por rotações e reviramentos em torno do centro. Uma rotação dada por uma transformação linear dos $x$ da forma:

$$
\left\{\begin{array}{l}
\rho x_{1}=a_{11} x_{1}^{\prime}+a_{12} x_{2}^{\prime}+a_{13} x_{3}^{\prime} \\
\rho x_{2}=a_{21} x_{1}^{\prime} a_{22} x_{2}^{\prime}+a_{23} x_{3}^{\prime} \\
\rho x_{3}=a_{31} x_{1}^{\prime}+a_{32} x_{2}^{\prime}+a_{33} x_{3}^{\prime} \\
\rho x_{4}=x_{4}^{\prime}
\end{array}\right.
$$


A matriz $\left(a_{k \ell}\right)$ é real e unitária. Os seus coeficientes podem ser representados parametricamente em função dos elementos de uma matriz binária unitária pelas fórmulas de Olinde Rodrigues:

$$
\begin{aligned}
& \begin{cases}a_{11}+i a_{21}=\alpha^{2}-\beta^{2} & a_{31}=\alpha \bar{\beta}-\beta \bar{\alpha} \\
a_{12}+i a_{22}=i\left(\alpha^{2}+\beta^{2}\right) & a_{32}=i(\alpha \beta-\beta \bar{\alpha}) \\
a_{13}+i a_{23}=-2 \alpha \beta & a_{33}=\alpha \bar{\alpha}-\beta \bar{\beta}\end{cases} \\
& \left\{\begin{array}{l}
\alpha \beta-\gamma \delta=1 \\
\alpha=\bar{\delta} \quad \beta=-\bar{\gamma}
\end{array}\right. \\
& \left\{\begin{array}{l}
\rho x_{1}=1 / 2\left(\alpha^{2}+\delta^{2}-\beta^{2}-\gamma^{2}\right) x_{1}^{\prime}+i / 2\left(\alpha^{2}-\delta^{2}+\beta^{2}+\gamma^{2}\right) x_{2}^{\prime}+(\alpha \bar{\gamma}+\gamma \bar{\alpha}) x_{3}^{\prime} \\
\rho x_{2}=i / 2\left(\delta^{2}-\alpha^{2}+\beta^{2}-\gamma^{2}\right) x_{1}^{\prime}+1 / 2\left(\alpha^{2}+\beta^{2}+\gamma^{2}+\delta^{2}\right) x_{2}^{\prime}+i(\gamma \bar{\alpha}-\alpha \bar{\gamma}) x_{3}^{\prime} \\
\rho x_{3}=(\alpha \bar{\beta}+\beta \bar{\alpha}) x_{1}^{\prime}+i(\alpha \bar{\beta}-\beta \bar{\alpha}) x_{2}^{\prime}+(\alpha \bar{\alpha}-\gamma \bar{\gamma}) x_{3}^{\prime} \\
\rho x_{4}=x_{4}^{\prime}
\end{array}\right.
\end{aligned}
$$

Ora se representarmos as coordenadas pelas fórmulas paramétricas (8) e efetuarmos sobre os sistemas de geratrizes uma projetividade de matrizes $\left(\begin{array}{ll}\alpha & \beta \\ \gamma & \delta\end{array}\right)$ e $\left(\begin{array}{cc}\bar{a} & \bar{\beta} \\ \bar{\gamma} & \bar{\delta}\end{array}\right)$ tornamos a obter as fórmulas (13). Podemos portanto enunciar o teorema:

"As rotações em torno do centro induzem, nos dois sistemas de geratrizes homografias conjugadas unitárias, e reciprocamente".

Um reviramento é o produto de uma simetria por um deslocamento. Uma simetria em relação à origem troca entre si os dois sistemas de geratrizes e portanto qualquer reviramento em torno da origem induz, sobre a esfera, uma projetividade de segunda espécie com as matrizes $\left(\begin{array}{ll}\alpha & \beta \\ \gamma & \delta\end{array}\right)$ e $\left(\begin{array}{ll}a & b \\ c & d\end{array}\right)$ unitárias e conjugadas. 


\section{Projetividades sobre uma esfera complexa}

4. As projetividades sobre uma esfera complexa podem ser tratadas de modo análogo ao usado no caso da esfera real. A diferença essencial entre os dois casos é que no caso da esfera real as duas famílias de geratrizes são imaginárias conjugadas ao passo que no caso da esfera complexa cada sistema é autoconjugado de modo que duas geratrizes de sistemas diferentes não podem se encontrar em pontos reais. Daí resulta serem independentes as homografias sobre os dois sistemas de geratrizes.

A equação da esfera complexa do raio - 1 com centro na origem é:

$$
x_{1}^{2}+x_{2}^{2}+x_{4}^{2}+x_{4}^{2}=0 \quad \text {. }
$$

Os dois sistemas de geratrizes tem por equações:

$$
\begin{gathered}
\left\{\begin{array}{l}
\lambda_{1}\left(x_{1}-i x_{2}\right)+\lambda_{2}\left(x_{3}-i x_{4}\right)=0 \\
\lambda_{1}\left(x_{3}+i x_{4}\right)-\lambda_{2}\left(x_{1}+i x_{2}\right)=0
\end{array}\right. \\
\left\{\begin{array}{l}
\mu_{1}\left(x_{1}-i x_{2}\right)+\mu_{2}\left(x_{3}+i x_{4}\right)=0 \\
\mu_{1}\left(x_{3}-i x_{4}\right)-\mu_{2}\left(x_{1}+i x_{2}\right)=0
\end{array}\right.
\end{gathered}
$$

As coordenadas podem ser representadas parametricamente do modo seguinte:

$$
\left\{\begin{array}{l}
\rho x_{1}=\lambda_{1} \mu_{1}-\lambda_{2} \mu_{2} \\
\rho x_{2}=-i\left(\lambda_{1} \mu_{1}+\lambda_{2} \mu_{2}\right) \\
\rho x_{3}=\lambda_{1} \mu_{2}+\lambda_{2} \mu_{1} \\
\rho x_{4}=i\left(\lambda_{1} \mu_{2}-\lambda_{2} \mu_{1}\right)
\end{array}\right.
$$


Este artigo publicado em Anais da Academia Brasileira de Ciências Vol. XIII, N.2, 30 junho 1941, pp.129-135, continua e conclue em "Sobre uma extensão do cálculo espinorial (II)", Anais da Academia Brasileira de Ciências, Vol. XV, N.2, pp.97-108, Rio de Janeiro, 30 de junho de 1943, que tem o seguinte:

\section{Sumário}

As relações entre os espinores e as projetividades sobre o absoluto são estudadas no caso de espaços euclidianos e não euclidianos com um número qualquer de dimensões. O conceito de representação espinorial é extendido a uma categoria de grupos que compreendem todos os que foram considerados na parte I. 